\title{
Impaired Endothelium-Mesenchymal Stem Cells Cross-talk in Systemic Sclerosis: a Link Between Vascular and Fibrotic Features
}

\author{
Paola Cipriani ${ }^{*}$, Paola Di Benedetto ${ }^{1}$, Piero Ruscitti ${ }^{1}$, Antonio Francesco Campese ${ }^{2}$, Vasiliki Liakouli ${ }^{1}$, \\ Francesco Carubbi ${ }^{1}$, Ilenia Pantano ${ }^{1}$, Onorina Berardicurt ${ }^{1}$, Isabella Screpanti ${ }^{2}$ and Roberto Giacomelli ${ }^{1}$
}

\begin{abstract}
Introduction: To assess if an impaired cross-talk between endothelial cells (ECs) and perivascular/multipotent mesenchymal stem cells (MSCs) might induce a perturbation of vascular repair and leading to a phenotypic switch of MSC toward myofibroblast in Systemic Sclerosis (SSC).

Methods: We investigated different angiogenic and profibrotic molecules in a tridimentional matrigel assay, performing co-cultures with endothelial cells (ECs) and bone marrow derived MSCs from patients and healthy controls (HC). After 48 hours of co-culture, cells were sorted and analyzed for mRNA and protein expression.

Results: ECS-SSC showed a decreased tube formation ability which is not improved by co-cultures with different MSCs. After sorting, we showed: i. an increased production of vascular endothelial growth factor A (VEGF-A) in SSC-MSCS when co-cultured with SSC-ECs; ii. an increased level of transforming growth factor beta (TGF- $\beta$ ) and platelet growth factor BB (PDGF-BB) in SSc-ECs when co-cultured with both HC- and SSc-MSCs; iii. an increase of TGF- $\beta$, PDGF-R, alpha smooth muscle actin (a-SMA) and collagen 1 (Col1) in both HC- and SSC-MSCs when cO-cultured with SSC-ECS.
\end{abstract}

Conclusion: We showed that during SSC, the ECs-MSCs crosstalk resulted in an altered expression of different molecules involved in the angiogenic processes, and mainly SSc-ECs seem to modulate the phenotypic switch of perivascular MSCs toward a myofibroblast population, thus supporting the fibrotic process.

\section{Introduction}

Systemic slcerosis (SSc) is an autoimmune disease characterized by early vascular abnormalities and subsequent fibroblass activation to myofibroblasts, leading to fibrosis of the skin and internal organs [1,2]. Much effort has been made in recent years, to identify the earlier clinical markers of SSc, to diagnose patients in a very early phase before the occurrence of fibrosis [3]. Similarly, much effort has been made to identify the earlier pathogenetic steps that may be able to activate fibroblasts after the endothelial injury [4]. Recently, many studies have pointed out the role of endothelial cells/ pericytes interactions in leading fibrosis after vascular

\footnotetext{
* Correspondence: paola.cipriani@cc.univaq.it

'Department of Applied Clinical Sciences and Biotechnology, Rheumatology Unit, School of Medicine, University of L'Aquila, Delta 6 Building, Via dell'Ospedale, 67100 L'Aquila, Italy

Full list of author information is available at the end of the article
}

injury, during different fibrotic disorders [5,6], and these models open new perspectives to understand the fibrotic process in SSc. At present, it is well-established that at the onset of the disease a microvascular injury, associated to both ischemia/reperfusion damage and impaired compensatory angiogenesis $[7,8]$, results in a capillary rarefaction with consequent tissue ischemia. The complex link between vessel disappearance and fibroblast activation is still largely unknown. It is well-known that the maintenance of the existing vasculature and the stabilization of the newly formed vessel is successfully achieved by strict interplay between endothelial cells (ECs) and pericytes, mediated by both direct contact and orchestrated actions of cytokines and growth factors [9].

During the normal angiogenic process, mesenchymal pericyte progenitors are recruited in the vascular sprouts in response to growth factors, mainly PDGF, which is 
produced by ECs [10]. The contact between ECs and perivascular progenitors is a pivotal step for inducing ECs to produce active transforming growth factor- $\beta$ (TGF- $\beta$ ) [11], which is able to induce maturation of mural cells $[12,13]$, promoting blood vessel stabilization and function [14]. Finally, vascular endothelial growth factor (VEGF), produced by mesenchymal stem cells (MSCs), makes ECs unresponsive to other proangiogenic stimuli.

Several studies provide evidence that, during adult life, resident pericytes, distributed in different tissues, represent the local sources of stem cells. In fact pericytes may differentiate toward a variety of mesenchymal populations, such as osteoblasts, chondrocytes, adipocytes and fibroblasts [15], supporting the concept that adult multipotent MSCs are members of the pericyte family and reside in a specialized physical location known as the perivascular niche [16]. In this place, MSCs regulate their own fate by the heterotypic cell-cell crosstalk with ECs [17]. This contact maintains MSCs in their quiescent state or, alternatively, provides signals activating their differentiative process [18]. This phenotypic modulation is a critical event, which may lead to myofibroblast differentiation, resulting in abnormal expression of contractile proteins, such as alpha-smooth muscle actin ( $\alpha$-SMA), and in collagen 1 (Col1) production. The pericyte-myofibroblast transition seems to be involved in many different diseases. As shown in experimental models of kidney fibrosis, altered pericyte-EC crosstalk, after microvascular injury leads to pericyte detachment, migration, and differentiation toward myofibroblasts with consequent destabilization and loss of capillaries $[19,20]$. Furthermore, in different experimental models, spinal cord pericytes are identified as the main progenitor population of scar tissue in the central nervous system, intestinal pericytes are the main source of myofibroblasts, in different models of colitis, and hepatic stellate cells are the major precursors of myofibroblasts in liver disease [21-23].

Recently, different authors have shown that bone marrow-derived MSCs express pericyte markers and cooperate with ECs to form a vascular network in threedimensional cultures, suggesting that these cells may derive from the bone marrow perivascular sites where they probably act as pericytes [24,25]. From a translational point of view, the data obtained in these models on the role of perivascular cells in the fibrotic process and their possible involvement in the pathogenesis of SSc, are intriguing and until now are largely unexplored.

We recently showed that MSCs from SSc patients express pericyte-specific markers and behave as pericytes. They display an antiangiogenic and myofibroblast-like phenotype, with increased production of contractile fiberassociated proteins, probably due to micro-environmental cues operating during the disease [26].
During SSc, the fibrotic process is strongly mediated by the effect of several cytokines, including TGF- $\beta$ and platelet-derived growth factor (PDGF), and elevated levels of VEGF have been reported, despite lack of compensative angiogenesis. Of note, it is well-known that the same molecules are involved in vessel formation and stabilization. Thus, a perturbation of the normal crosstalk between ECs and perivascular/MSCs in SSc might induce an abnormal production of these molecules, inducing vessel instability and perivascular-myofibroblast phenotypic transition and activation, starting the pathogenic process leading to the classical hallmarks of the disease: the diffuse fibrosis, on one hand, and the loss of peripheral vascularization on the other. The aim of the present study was to assess these specific molecular pathways during the EC-MSC crosstalk in patients with early diffuse SSc, in whom the occurrence of fibrosis is generally earlier and rapidly progressive, when compared to the limited form. We performed a three-dimensional angiogenic assay, co-culturing ECs and MSCs from SSc patients and healthy controls (HCs). After co-culture, cells were sorted for molecular- and protein-level analyses. This is the first report in the scientific literature providing evidence that ECs in SSc may induce a pathogenic phenotype in MSCs, switching from architectural and metabolic supporting cells to cells with strong migratory and profibrotic functions, thus potentially modulating vessel instability on one hand, and different pathways involved in the fibrotic process on the other hand.

\section{Methods}

\section{Isolation, culture, immunophenotyping and} differentiation of MSCs

After approval from San Salvatore University Hospital ethics committee and written informed consent from patients, MSCs were obtained from 10 SSc patients with the early diffuse cutaneous form of the disease (disease duration less than 3 years calculated since the first nonRaynaud's symptom of SSc) [27], by aspiration from the posterior superior iliac crest and cultured and characterized as previously described [28]. Demographic and clinical characteristics of the patients are shown in Table 1.

Patients discontinued corticosteroids, oral vasodilators, intravenous prostanoids or other potentially diseasemodifying drugs, at least one month before biopsies. None were assumed to be taking immunosuppressants.

Ten frozen bone marrow (BM)-MSCs samples obtained from age-matched healthy women who were bone marrow donors were used as control. MSCs from both SSc patients and $\mathrm{HCs}$ were plated at a concentration of $2 \times 10^{5}$ cells $/ \mathrm{cm}^{2}$ in D-MEM (GIBCO, Carsbald, CA, USA) supplemented with 10\% FBS (Standard South 
Table 1 Clinical and demographic features of the 10 patients with early diffuse SSc

\begin{tabular}{lllllll}
\hline $\begin{array}{l}\text { Sex/age } \\
\text { (years) }\end{array}$ & $\begin{array}{l}\text { Year of SSc onset/disease } \\
\text { duration at skin-BM } \\
\text { biopsy (years) }\end{array}$ & $\begin{array}{l}\text { MRSS/score } \\
\text { at skin biopsy }\end{array}$ & Autoantibodies & $\begin{array}{l}\text { Lung } \\
\text { involvment } \\
\text { HRCT/PFT }\end{array}$ & $\begin{array}{l}\text { Heart involvement/ } \\
\text { scleroderma renal } \\
\text { crisis }\end{array}$ & $\begin{array}{l}\text { Raynaud's } \\
\text { phenomenon/ } \\
\text { digital ulcers }\end{array}$ \\
\hline F/46 & $2010 / 2$ & $12 / 2$ & ANA/Scl-70 & Normal/Normal & Normal/No & Yes/No \\
F/21 & $2009 / 3$ & $13 / 1$ & ANA/Scl-70 & Normal/Normal & Normal/No & Yes/Yes \\
F/31 & $2011 / 1$ & $13 / 2$ & ANA/Scl7-0 & Normal/Normal & Normal/No & Yes/Yes \\
F/36 & $2010 / 2$ & $11 / 2$ & ANA/Scl-70 & Normal/Normal & PAH/No & Yes/No \\
M/20 & $2010 / 2$ & $11 / 1$ & ANA/Scl-70 & Normal/Normal & Normal/No & No/No \\
F/41 & $2010 / 2$ & $15 / 2$ & ANA/Scl-70 & Normal/Normal & Normal/No & Yes/No \\
F/30 & $2010 / 2$ & $10 / 1$ & ANA/Scl-70 & Normal/Normal & Normal/No & Yes/No \\
F/21 & $2010 / 2$ & $09 / 1$ & ANA/Scl-70 & Normal/Normal & Normal/No & Yes/No \\
F/31 & $2009 / 3$ & $14 / 1$ & ANA/Scl-70 & Normal/Normal & Normal/No & Fes/No \\
F/42 & $2009 / 3$ & $16 / 2$ & ANA/Scl-70 & Fibrosis/Normal & Normal/No & \\
\hline
\end{tabular}

M, male; F, female; MRSS, modified Rodnan skin thickness score (maximum possible score 51); HRCT, high-resolution computed tomography; PFT, pulmonary function test; ANA, antinuclear antibodies; ACA, anti-centromere antibodies; scl-70, anti-topoisomerase antibodies; PAH, pulmonary arterial hypertension.

America origin, Lonza, MD, USA), $2 \mathrm{mmol} / \mathrm{L}$ L-glutamine (EuroClone, Milano, Italy) and $100 \mathrm{U}$ Penicillin, 1,000 U Streptomycin (Biochrom AG, Miramar, Fl, USA). At 80\% confluence the BM-MSCs were split and sub-cultured. Third-passage (P3) BM-MSCs were analyzed for the surface expression of MSCs antigens (CD45, CD73, CD90, CD34, CD79a, PDGFR $\beta$ ) and pericyte markers $(\alpha$-SMA, SM22 $\alpha$, NG2, desmin, RGS5) as previously described [24].

\section{Endothelial cell isolation and culture}

Microvascular ECs were isolated from skin biopsies from the same 10 SSc patients and the same 10 bone marrow donors, who kindly offered a skin sample for research purposes, after written informed consent. Biopsy samples $(1 \times 0.5 \mathrm{~cm})$ of the involved forearm skin (skin score $1 / 2$ at the biopsy site) were washed with PBS (Life Technologies, Van Allen Way Carlsbad, CA, USA) and four explants were placed into a 50-mL tube containing $15 \mathrm{~mL}$ of trypsin (Sigma-Aldrich, MO, USA) and then to digest for 45 minutes at $37^{\circ} \mathrm{C}$. Cells were cultured in EGM2-MV (Lonza, Walkersville, MD, USA) at $37^{\circ} \mathrm{C}$ in a humidified atmosphere of $5 \% \mathrm{CO} 2$.

Before the cells reached confluence after approximately 1 week, the heterogeneous pool of cells was exposed to a CD31-positive selection, performed with the Dynabeads magnetic CD31 MicroBeads cell sorting system (Invitrogen, Life Technologies, CA, USA). The beads rapidly target and partially coat the endothelial cells expressing the CD31 receptor.

After the incubation, the cells were placed in a magnet (Dynal MPC-S) (Invitrogen, Life Technologies, CA, USA) for 2 minutes, following the manufacturer's recommended protocol for washing and final extraction. The CD31negative cells (with no beads attached) were removed during the successive washings. The positive selected cells were $99 \%$ ECs with a specific phenotype (CD-31, CD-34, CD-144) (Figure 1a). The cells were used at the third passages.

\section{In vitro angiogenesis assay}

Tube formation ability was evaluated using a Matrigel assay (Matrigel (BD Biosciences, Qume Drive San Jose, CA, USA) $(8.6 \mathrm{mg} / \mathrm{mL})$ was used at 1:1 dilution with EGM2MV, without supplement. ECs and MSCs were labeled, before co-culture in Matrigel, using the green fluorescent dye PKH67 and red fluorescent dye PKH26 (Sigma, St. Louis, MO, USA), respectively, according to the manufacturer's instructions. ECs and MSCs were seeded alone and in coculture ECs/MSCs in a 3:1 ratio. The following co-cultures were performed: SSc-ECs/SSc-MSCs, SSc-ECs/HC-MSCs, HC-ECs/HC-MSCs, HC-ECs/SSc-MSCs. After 48 hrs the total tube length of each well was measured as cells $/ \mathrm{mm}$ and photographed. Images were acquired using an Olympus BX53 fluorescence microscope.

\section{Cell sorting of co-cultured cells}

After $48 \mathrm{hrs}$ of co-culture, as suggested by available literature [29,30], tube formation was observed. At this point, MSCs and ECs were gently removed from three-dimensional cultures, using Dispase (BD Biosciences Discovery Labware, Bedford, MA, USA), and mixed with $5 \mathrm{mM}$ ethylenediaminetetraacetic acid (EDTA). Dispase effectively degrades Matrigel without damaging the embedded cells. Both recovered MSCs and ECs were washed several times with PBS and re-suspended in 0.1\% FBS in PBS for cell-sorting. The cells were sorted (purity >95\%) by a FACSAria cell sorter (BD Biosciences, Franklin Lakes, NJ, USA) and further used for gene expression and protein profiling. 


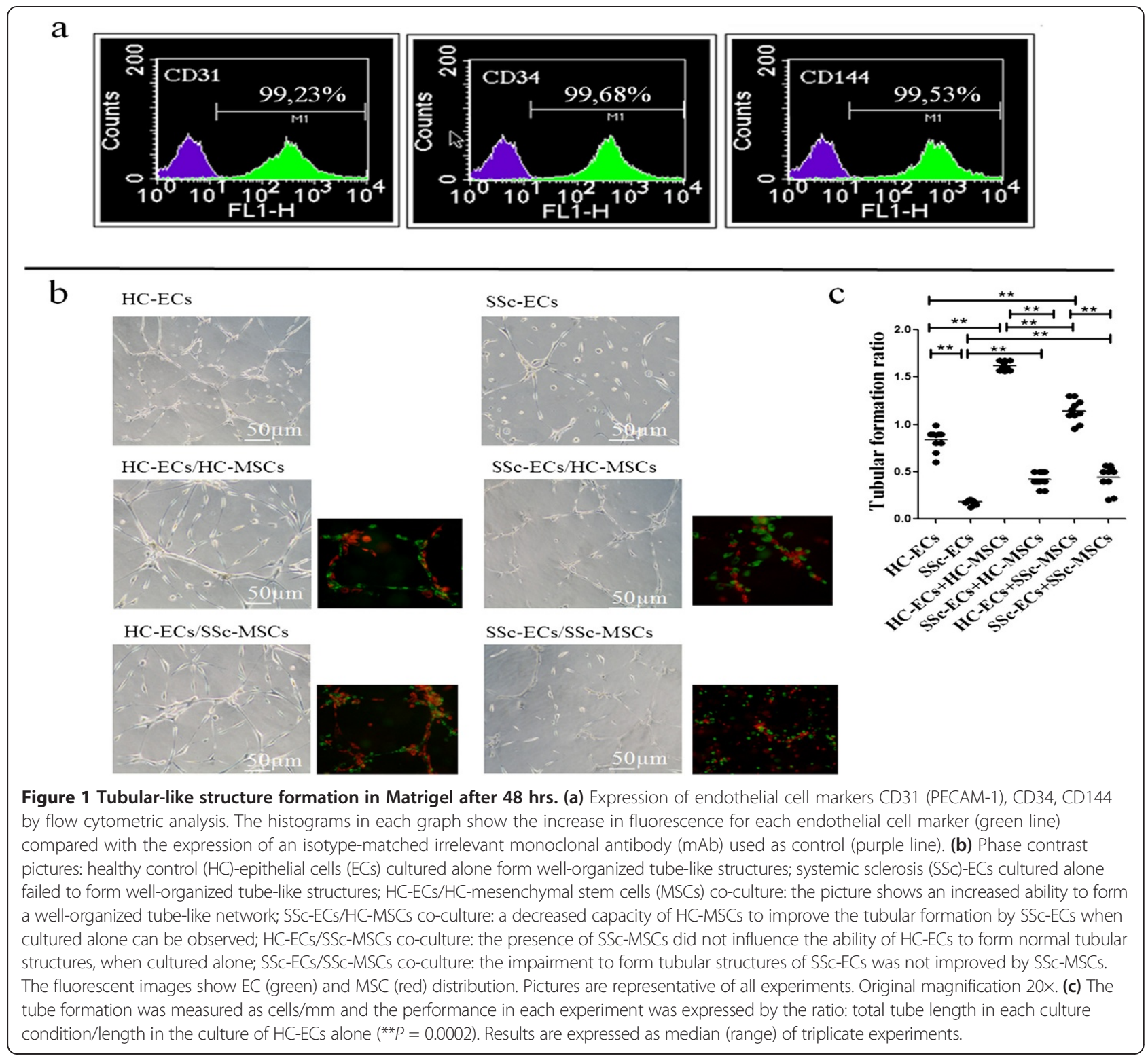

\section{qRT-PCR analysis}

Total RNA was extracted from sorted BM-MSCs and ECs using NucleoSpin RNAXS (Macherey Nagel, Dueren, Germany) according to manufacturer's instructions and reverse-transcribed into complementary DNA (cDNA) with the ThermoScript reverse-transcription PCR system (Invitrogen, Life Technologies, CA, USA). The qRT-PCR was performed using SYBR green kits (Applied Biosystems, Life Technologies, Van Allen Way Carlsbad, CA, USA). Results were analyzed after 45 cycles of amplification using the ABI 7500 Fast Real Time PCR System. Primers were designed on the basis of the reported sequences (Primer bank NCBI; $\beta$-actin: 5 '-CCTGGCAC CCAGCACAAT-3' (forward) and 5' -AGTACTCCGTGT GGATCGGC-3' (reverse); $\alpha$-SMA:5' -CGGTGCTGTCTC
TCTATGCC-3' (forward) and 5' -CGCTCAGTCAGGAT CTTCA-3' (reverse); VEGF-A: 5'-AGGGCAGAATCAT CACGAAGT-3' (forward) and 5'-GCTGCGCTGATAGA CATCCA-3' (reverse); VEGFR2:5'-GTGATCGGAAATG ACACTGGAG-3' (forward) and 5' - CATGTTGGTCAC TAACAGAAGCA-3' (reverse); Col1A1: 5'-AGGGCCA AGACGAAGACAGT-3' (forward) and 5' -AGATCACG TCATCGCACAACA-3' (reverse); Col1A2: 5' -TCTGGAT GGATTGAAGGGACA-3' (forward) and 5' - CCAACAC GTCCTCTCTCACC-3' (reverse); TGF- $\beta$ : 5' -CTAATGGT GGAAACCCACAACG-3' (forward) and 5' - TATCGCCA GGAATTGTTGCTG-3' (reverse); PDGF-RR: 5' -TGAGCG GAAACGGCTCTAC-3' (forward) and 5' - AGTTCCTCG GCATCATTAGGG-3' (reverse); PDGF-BB: $5^{\prime}$-CTCGAT CCGCTCCTTTGATGA-3' (forward) and 5' - CGTTGGT 
GCGGTCTATGAG-3' (reverse). All gene expression data were normalized to those for $\beta$-actin.

\section{Western blot}

In order to perform western blot assays, sorted BM-MSCs and ECs cells were pelleted, washed twice with PBS, lysed on ice in lysis buffer (1\% Triton X-100, 0.5\% NP-40, 50mMTris-Cl, pH 7.5, $150 \mathrm{mMNaCl}, 1 \mathrm{mM}$ EDTA, supplemented with $1 \mathrm{mMphenylmethylsulfonyl} \mathrm{fluoride,} 1$ mMNaF, $1 \mathrm{mM} \mathrm{Na} \mathrm{VO}_{4}, 5 \mu \mathrm{g} / \mathrm{mL}$ aprotinin, $5 \mu \mathrm{g} / \mathrm{mL}$ leupeptin) for 30 minutes and cleared by centrifugation. The protein concentration was calculated by Bradford protein assay reagent (Bio-Rad Laboratories S.r.l., Segrate, MI, Italy): $50 \mu \mathrm{g}$ of proteins were separated by SDS-PAGE and transferred to nitrocellulose membranes. After $1 \mathrm{hr}$ at room temperature in blocking buffer ( $5 \%$ non- fat milk in Tris-buffered saline/1\% tween 20 (TBS/T)) the membranes were washed three times for 5 minutes each in $\mathrm{TBS} / \mathrm{T}$, and incubated overnight at $4^{\circ} \mathrm{C}$ with the primary antibodies: VEGFR-2 (Invitrogen Corporation, CA, USA), VEGF-A, PDGF-BB and TGF- $\beta$ (Abcam, MA, USA), PDGF-R, Col1A1 and $\alpha$-SMA (Santa Cruz, Biotechnology, Dallas, Texas, USA), diluted in 5\% bovine serum albumin in TBS/ T. Following three washes with TBS/T, horseradish peroxidase-conjugated secondary antibodies (Santa Cruz, Biotechnology) diluted in blocking buffer was added for 30 minutes at room temperature and washed three times with TBS/T. The detection was performed by enhanced chemiluminescence detection ECL reaction (Amersham Pharmacia Biotechnology Piscataway, NJ, USA). All the signals were quantified by normalizing to the tubulin signal (CP06 Anti- $\alpha$-Tubulin Mouse mAb -DM1A). The levels of proteins, in HC-MSCs cultured alone, were set to $100 \%$; and all the results were normalized to this value. Immunoreactive bands were quantified with densitometry using Image software (NIH, Bethesda, MD, USA).

\section{Cytofluorimetric analysis}

After sorting, HC- and SSc-MSCs were incubated with the following conjugated monoclonal antibodies: allophycocyanin (APC)-conjugated monoclonal antibodies, including anti-human CD140b (PDGFRb) (BioLegend, San Diego, CA, USA) and anti-human CD309 (VEGFR2) (Miltenyi Biotec, San Diego, CA, USA ). Each fluorescence analysis included appropriate APC-conjugated negative isotype controls.

\section{Enzyme-linked immunosorbent assay}

The concentration of VEGF-A, TGF- $\beta$ and PDGF-BB released in single cultures and in co-culture supernatants were determined by ELISA using Quantikine Human Immunoassay kits (all by R\&D Systems, Minneapolis, MN, USA), according to the manufacturer's protocol. After $48 \mathrm{hrs}$ of co-culture and tube formation, media samples were collected and tested.

\section{Statistical analysis}

GraphPad Prism 5.0 software was used for statistical analyses. Results are expressed as median (range). Due to the non-parametric distribution of our data the MannWhitney $U$-test was used as appropriate for analyses. Statistical significance was expressed by a $P$-value $\leq 0.05$.

\section{Results}

SSc-ECs affect the MSCs skill to support tubulogenesis

In our in vitro matrigel assay HC-ECs cultured alone formed organized tube-like structures. When these cells were co-cultured with $\mathrm{HC}$ - and SSc-MSCs, we observed a significant improvement in tube-formation ability without a statistically significant difference. On the contrary, as shown in Figure 1b-c, using SSc-ECs a significant impairment in tube formation was observed.

Impaired production of angiogenic and fibrotic molecules in SSC-ECs and SSc-MSCs sorted after $48 \mathrm{hrs}$ of co-culture VEGF-A/VEGFR2 expression in MSCs

When MSCs were cultured alone, a statistically significant increase in VEGF-A transcript levels was observed in SScMSCs compared to HC-MSCs (2.11 (1.76 to 3.10) versus 1.11 (0.80 to 1.46$)$, respectively, $P<0.0001)$. As shown in Figure $2 \mathrm{a}$ the presence of SSc-ECs strongly increase the levels of VEGF-A mRNA transcript in SSc-MSCs when compared to the other possible mixture of cells $(P<0.0001$ for all conditions). Basal transcript levels of VEGFR2 were significantly higher in SSc-MSCs than in HC-MSCs (1.54 (1.10 to 2.09 ) versus 1.28 (1.00 to 1.36 ), respectively, $P<0.0088)$. The VEGFR2 mRNA levels were significantly upregulated after co-culture with ECs when compared to basal conditions ( $P \leq 0.0002$ for both). Mirroring what was observed for the VEGF-A, the expression of VEGFR2 was strongly increased in SSc-MSCs when co-cultured with SSc-ECs, as shown in Figure $2 \mathrm{~b}$. These results were confirmed at the protein level by western blotting analyses (Figure 2c). As far as the surface expression of VEGFR2 is concerned, as shown in Figure 2e, we observed a strong increase in intensity of VEGFR2 in SSc-MSCs when cocultured with SSc-ECs.

Furthermore, the VEGF-A protein secreted in each culture, mirrored the results observed by $\mathrm{qPCR}$ and western blot analysis (Figure 2d).

\section{SSC-ECs increase their expression of TGF- $\beta$ and PDGF-BB, after co-culture with MSCs}

We investigated the expression of TGF- $\beta$ in SSc-ECs and in SSc-MSCs cultured alone and after co-cultures. At the basal condition, the levels of TGF- $\beta$ in SSc-ECs were markedly increased when compared with HC-ECs $(3.18$ (2.58 to 3.76 ) versus 1.40 (0.91 to 1.98$)$, respectively, $P<0.0001)$. When SSc-ECs co-cultured, independent of the kind of MSCs, the highest levels of TGF- $\beta$ mRNA 


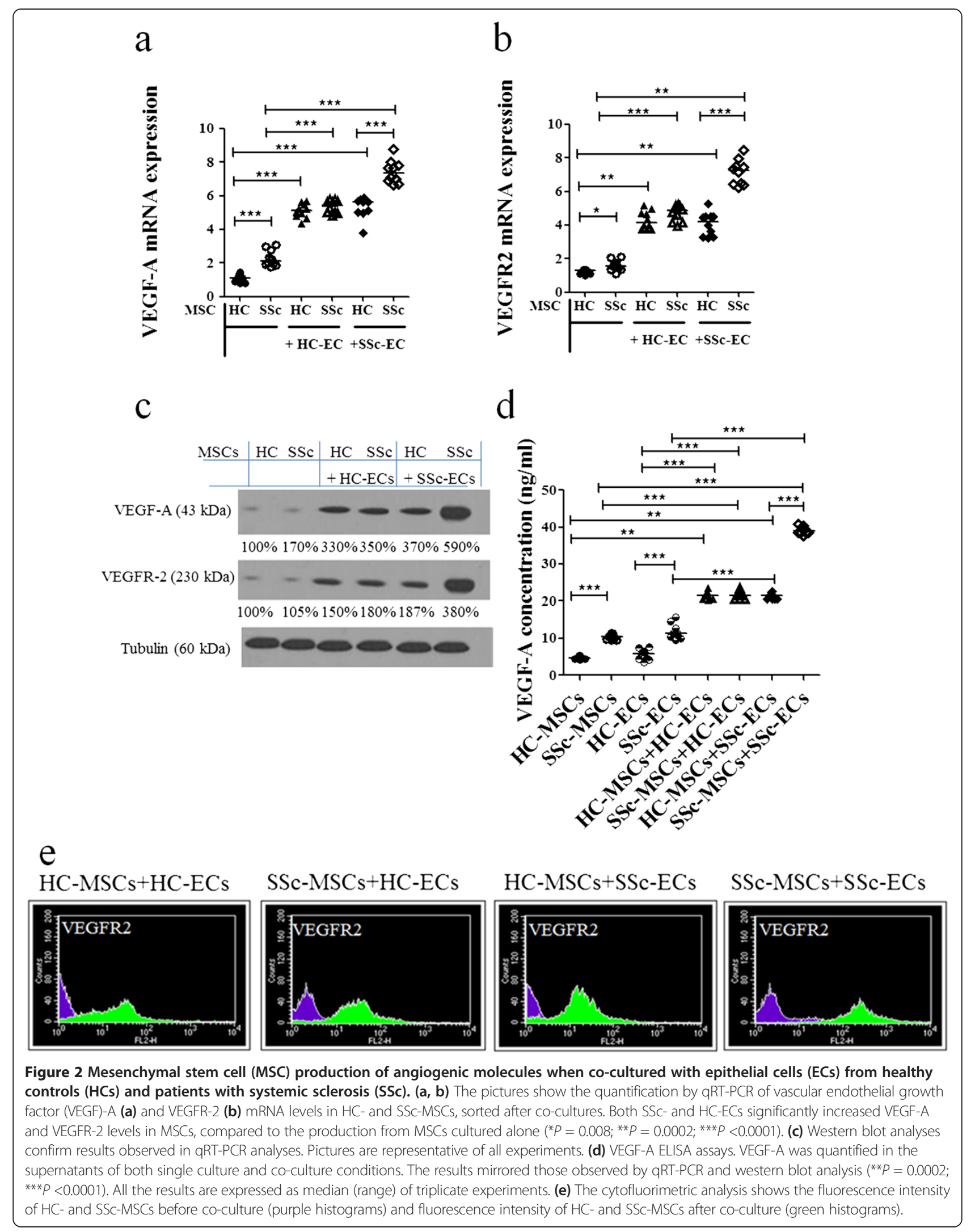


expression were observed, as shown in Figure 3a. These results were confirmed at the protein level by western blotting analyses (Figure $3 \mathrm{~b}$ ).

$P D G F-B B$ was markedly increased in SSc-ECs cultured alone when compared to HC-ECs (4.28 (2.58 to 4.98) versus 0.98 (0.87 to 1.57 ), respectively, $P<0.0001$ ]. Mirroring the results obtained for TGF- $\beta$ PDGF-BB levels were strongly increased in SSC-EC after any kind of coculture, as shown in Figure 3c; western blotting analysis confirmed these data (Figure $3 \mathrm{~b}$ ).

\section{SSc-MSCs increase their expression of TGF- $\beta$ and PDGF- $R$ after co-culture with ECs}

$T G F-\beta$ expression at the basal condition was similar in HC- and SSc-MSCs. As shown in Figure 4a, when SScECs were added to the culture, we observed the strongest increase of TGF- $\beta$ mRNA transcript levels in SSc-MSCs, when compared to the basal condition and when compared to the other possible mixture of cells $(P<0.0001)$.

No significant difference was observed in $P D G F-R$ mRNA transcripts levels between HC- and SSc-MSCs at the basal condition. We observed a significant upregulation after co-culture with ECs when compared to basal conditions. The expression of $P D G F-R$ was strongly increased in SSc-MSCs when co-cultured with SSc-ECs (Figure 4b).These results were confirmed at the protein level by western blotting analyses (Figure $4 d$ ). Similarl to what was observed for VEGFR2, the PDGF-R intensity of expression was strongly increased in SSc-MSCs when co-cultured with SSc-ECs (Figure 4c).

The TGF- $\beta$ and PDGF-BB quantification by ELISA showed increased release of the growth factors in the EC/MSC co-culture when compared with ECs and MSCs cultured alone (Figure 4e, f).
Impaired cross-talk between SSC-ECs and MSCs contribute to fibrotic switching in SSC-MSCs

Aiming to test whether the co-cultures of SSc-MSCs with ECs may contribute to SSc-MSC differentiation toward the myofibroblastic phenotype, we examined the levels of expression of $\alpha-S M A, \operatorname{Col1A1}$, and Col1A2 in both SSc- and HC-MSCs.

At the basal condition, SSc-MSCs showed a significant increase in mRNA levels of $\alpha-S M A$, Col1A1 and Col1A2 when compared with $\mathrm{HC}-\mathrm{MSCs}(\alpha-S M A$ transcript levels: SSc-MSCs 2.12 (1.92 to 2.40) versus HC-MSCs 1.09 (0.97 to 1.40$), P=0.0002$; Co1A1 transcript levels: SSc-MSCs 2.40 (1.80 to 3.20 ) versus $\mathrm{HC}$-MSCs 1.15 (0.97 to 1.40 ), $P=0.0002$; Col1A2 transcript levels: SSc-MSCs 2.55 (2.00 to 2.90 ) versus $\mathrm{HC}-\mathrm{MSCs} 0.80$ (0.60 to 1.20$), P=0.0002)$. After co-culture with $\mathrm{HC}$-ECs, the SSc-MSCs displayed significant downregulation of $\alpha-S M A$ and collagen gene expression when compared to basal levels ( $\alpha-S M A$, $P=0.0028$; Col1A1, $P=0.0002$; Col1A2, $P=0.0002)$, as shown in Figure 5a-c. After co-culture with SSc-ECs: 1) $\mathrm{HC}-\mathrm{MSC}$ showed a significant increase of $\alpha-S M A$ and collagen-related genes when compared to their basal levels ( $\alpha$-SMA, $P=0.0002$; Col1A1, $P=0.0002$; Col1A2, $P=0.0002)$; 2) SSc-MSCs did not show any difference in the expression of these genes when compared to their basal conditions. Finally, the western blot analyses of $\alpha$-SMA and Col1A1 mirrored the molecular results (Figure 5d).

\section{Discussion}

This descriptive study is the first report in the available literature, providing evidence that the pathologic endothelium, via altered MSC-EC crosstalk, impairs the angiogenic process, and modulate the production of a

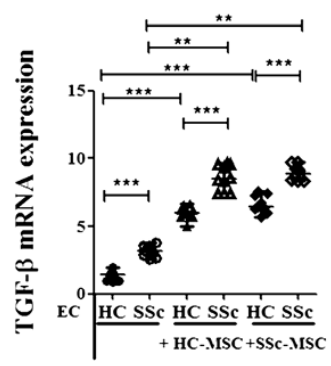

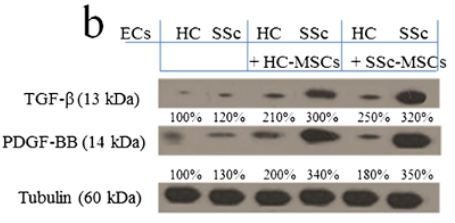

C

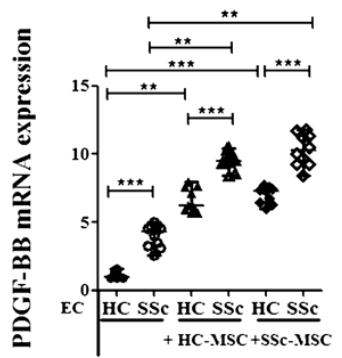

Figure 3 Epithelial cell (EC) production of angiogenic molecules when co-cultured with mesenchymal stem cells (MSCs) from healthy controls (HCs) and systemic sclerosis (SSc) patients. (a-c) Expression of transforming growth factor- $\beta$ (TGF- $\beta$ ) and platelet-derived growth factor (PDGF)-BB in ECs, before and after co-culture with MSCs. SSc-ECs showed a higher amount of mRNA expression levels of both TGF- $\beta$ (a) and PDGF-BB (c) compared to the expression levels of HC-ECS, when cultured alone. After co-culture with HC- and SSC-MSCS a significant increase of these expressions was observed, compared to the levels when ECs were cultured alone, the higher levels observed when SSc-ECs were in the co-cultures. All the results are expressed as median (range) of triplicate experiments ( ${ }^{* *} P=0.0002$; ${ }^{* * *} P<0.0001$ ). (b) Western blot analysis of TGF- $\beta$ and PDGF-R protein in ECs, confirmed those observed at molecular level Pictures are representative of all experiments. 


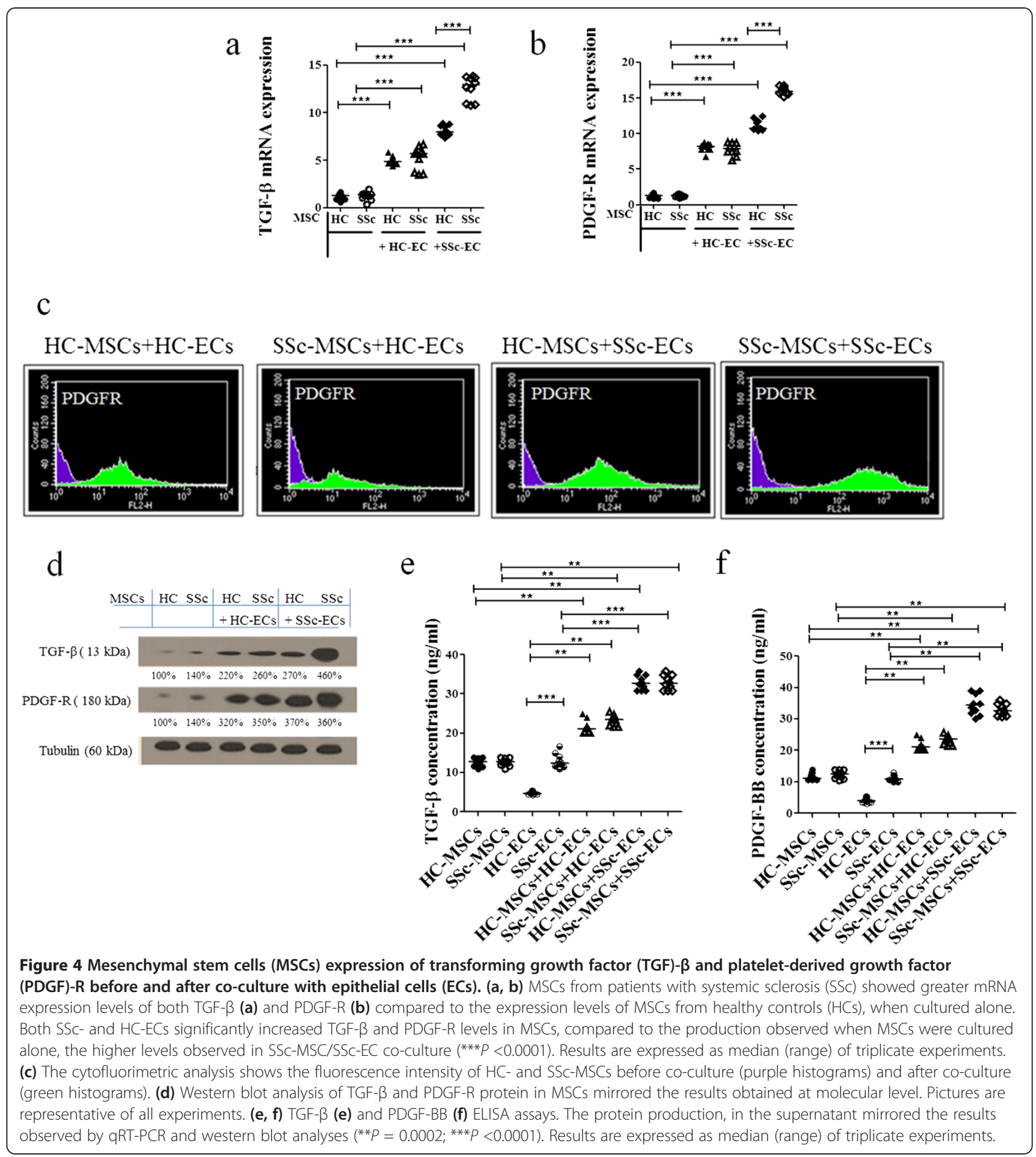

profibrotic molecules in both normal and pathologic MSCs, thus eliciting a phenotypic switch of these multipotent perivascular cells toward a myofibroblastic/profibrotic phenotype. Our data may support the hypothesis that in SSc, as shown in other pathological model of fibrosis [31], previously damaged ECs [32] may be involved in the earlier steps leading to fibrosis.
Using a tri-dimensional Matrigel system for EC/MSC co-cultures, we showed a highly significant reduction of the ability to form tubular structures when SSc-ECs were cultured with SSc-MSCs, whereas the presence of MSCs (from both patients and $\mathrm{HCs}$ ) in each condition improved the angiogenic ability of ECs cultured alone. To better understand this finding, after $48 \mathrm{hrs}$ of co- 

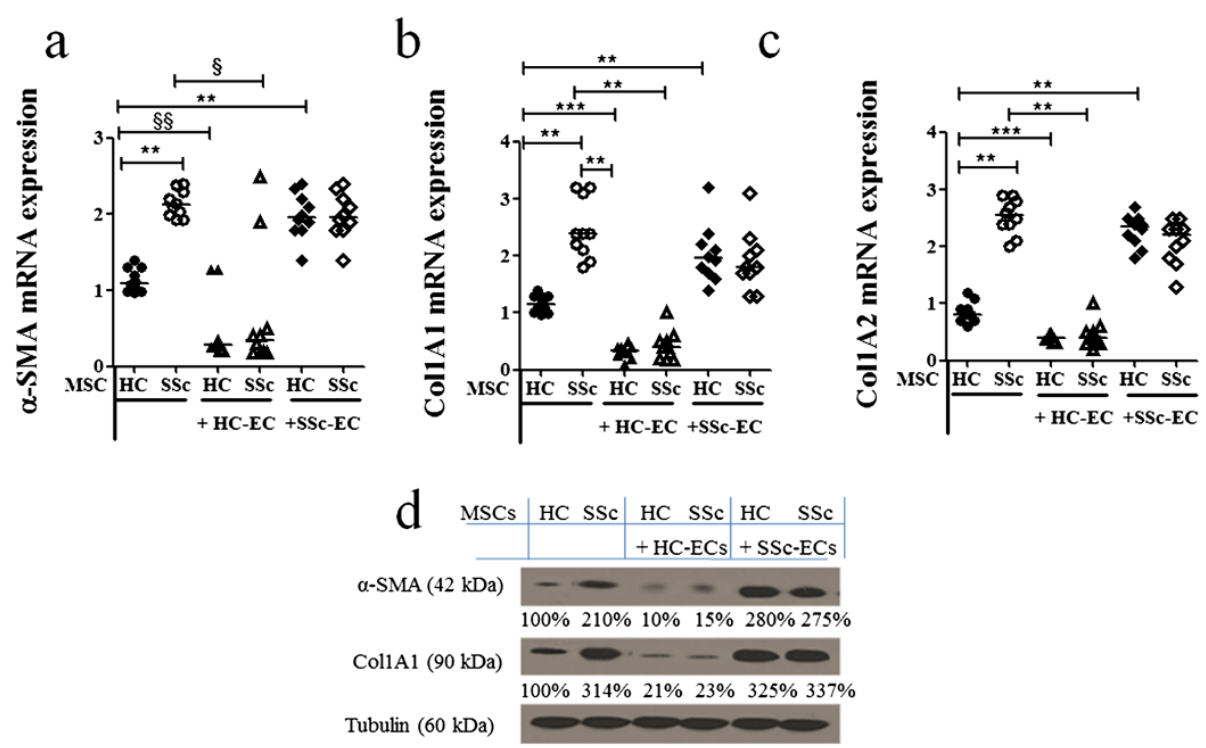

Figure 5 Mesenchymal stem cell (MSC) expression of myofibroblastic molecules before and after co-culture with epithelial cells (ECs). $(\mathbf{a}, \mathbf{c})$ When cultured alone, SSc-MSCS showed higher levels of alpha-smooth muscle actin (a-SMA) (a), collagen (Col)1A1 (b) and Col1A2 (c) compared to MSCs from healthy controls (HCs) cultured alone. Co-cultures with HC-ECs, significantly decreased the mRNA levels of these genes in HC- and MSCs from patients with systemic sclerosis (SSC). On the contrary, SSc-ECs did not downregulate these gene expressions, when cultured with both HC- and SSC-MSCS $\left({ }^{\S} P=0.0028 ;{ }^{\S \S} P=0.0073 ;{ }^{* *} P=0.0002 ;{ }^{* * *} P<0.0001\right)$. Results are expressed as median (range) of triplicate experiments. (d) Western blot analyses confirmed, at protein level, the results observed by qRT-PCR analyses. Pictures are representative of all experiments.

culture, we generated purified populations of cells by cell sorting. The analyses of cellular behavior after this interplay resulted in dramatic changes in gene expression and levels of proteins involved in the main steps of the angiogenic process, including the upregulation of profibrotic and extracellular matrix molecules.

In fact, as far as the production of VEGF-A, by MSCs, was concerned, the highest levels of gene expression and protein production were observed in SSc-MSCs when cocultured with SSc-ECs, although paradoxically the same co-culture showed the worst tube-formation performance. To understand this apparent paradox, it must be pointed out that as already shown [33], perivascular cell-derived VEGF-A exerts a unique role in driving blood vessel maturation and stability, functionally distinct from tissuederived VEGF-A, the latter promoting the vessel instability that precedes EC detachment and migration at the beginning of the angiogenic process. This physiologic switch, from tissue-derived to mural cell-derived VEGF-A, occurring at the end of the vessel maturation, fosters vessel stabilization and ultimately leads to tissue-derivedVEGF independence [33,34]. Furthermore, Greemberg et al. [35], demonstrated that VEGF abolished pericyte coverage of vascular sprouts, leading to vessel destabilization. VEGF-mediated activation of VEGFR2 suppressed PDGFR signaling, through the induction of a VEGFR/PDGFR complex. These data might explain our results of lower angiogenic performance, despite the increased VEGF-A production. Alternatively, a switch from proangiogenic to antiangiogenic VEGF isoforms might be considered, as recently described in SSc [36,37], although available literature shows conflicting results about the real presence and the potential inhibitory role of these antiangiogenic isoforms in human tissue [38].

Recent studies proposed an intriguing novel role for perivascular cells as a potential source of myofibroblasts in wound healing and in fibrotic disorders. Strong evidence has arisen from studies of the kidney, skin, lung, liver, and brain $[39,40]$. SSc, mirroring other fibrotic diseases, is characterized by the abnormal persistence of myofibroblasts, overexpressing the highly contractile protein $\alpha$-SMA and producing collagen and extracellular matrix components [31]. On the bases of our results, we may hypothesize that this myofibroblast differentiation reflects a phenotypic switch of multipotent perivascular cells in the disease. It has been suggested that these cells inside the vascular niche, which are considered a natural reservoir of MSCs/pericyte lineage, are activated to migrate and differentiate toward myofibroblasts, under the influence of ECs $[15,18]$. In this context, we described that SSc-ECs strongly increased the expression of $\alpha$-SMA and collagen-related genes, both in SSc- and HC-MSCs, which were already upregulated in SScMSCs. Of note, HC-ECs were able to downregulate both $\alpha$-SMA and collagen genes in SSc-MSCs. Taken together, these results show the pivotal role played by 
pathological ECs from SSc patients in influencing the micro-environment, by factors released during the crosstalk with pericytes, and point out the pivotal role of dysfunctional SSc-ECs in influencing MSC differentiation (derived from patients or controls) toward myofibroblasts. These in vitro data suggest the hypothesis that endothelial cell dysfunction, which is a very early event, in SSc patients, may be a potential trigger leading to fibrosis, via the involvement of multipotent perivascular cells.

We showed that SSc-ECs constitutively expressed higher amounts of PDGF-BB and TGF- $\beta$. Furthermore, when SSc-ECs were co-cultured with SSc-MSCs or $\mathrm{HC}-\mathrm{MSCs}$ they induced significantly increased production of both these molecules from MSCs. Recently, it has been shown that the overexpression of PDGF or TGF- $\beta$ in normal mouse skin, engineered by adenoviral vectors, leads to pericyte detachment and vessel destabilization [41]. Furthermore, PDGF and TGF- $\beta$ induced accumulation and expansion of connective tissue cells in the perivascular space, expressing both pericyte and fibroblast markers. Taken together, these data suggest that PDGF and TGF- $\beta$, produced by ECs play an important role in the generation and expansion of myofibroblasts, originating from microvascular pericytes [42].

Although PDGF and TGF- $\beta$ promote vessel stabilization via pericyte progenitor recruitment and maturation during vasculogenesis in embryonic life, the same molecules during adult life might play an opposite role in reactive conditions after vascular injuries, thus, leading to vessel destabilization and myofibroblast generation [43].

The interaction between TGF- $\beta$ and PDGF- $R$ has been recently shown as a unique characteristic in scleroderma fibroblasts, which respond to TGF- $\beta$ with upregulation of PDGF-R, making scleroderma fibroblasts more responsive to the subsequent mitogenic stimulation with PDGF [44]. In our experiments we observed constitutive upregulation of TGF- $\beta$ in SSc-ECs. These cells were able to induce strong upregulation of PDGF-R on SSc-MSCs, when co-cultured together. These findings support the hypothesis that an intramural PDGF/PDGF-R loop, synergistic with TGF- $\beta$ production, may cooperate in myofibroblast differentiation.

At present, emerging data suggest that MSCs are part of the perivascular niche, in which heterotypic cell-cell crosstalk between ECs and MSCs is able to regulate the perivascular cell fate [15]. This intimate interaction maintains MSCs in their quiescent state or provides signals toward differentiation, aiming to repair damaged tissues. Using a three-dimensional system for coculturing ECs and MSCs, followed by cell sorting, we demonstrated altered crosstalk between MSCs and ECs in SSc, resulting in pathogenetically relevant changes in gene expression and protein levels of several molecules involved in the angiogenic and profibrotic processes which are schematically represented in Figure 6. These alterations lead to a phenotypic switch of perivascular multipotent cells toward migratory and profibrotic

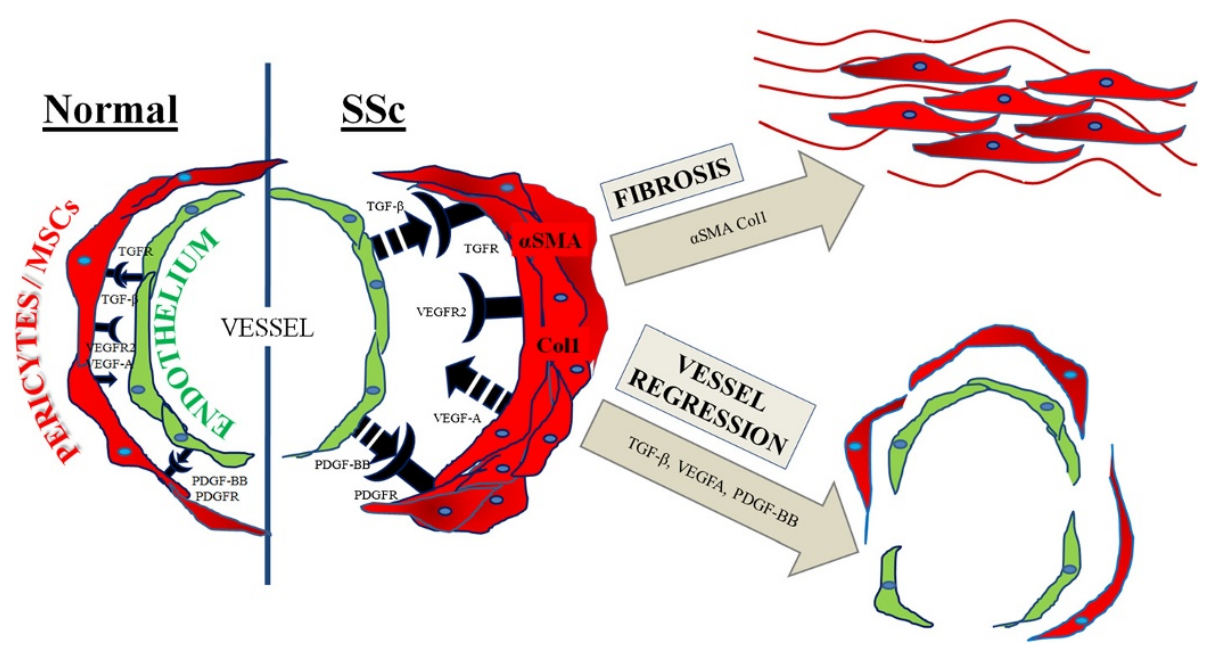

Figure 6 Schematic drawing that illustrates the pathogenic consequences of altered crosstalk between epithelial cells (ECs) and pericytes/mesenchymal stem cells (MSCs) in systemic sclerosis (SSc). In SSC, abnormal EC production of transforming growth factor (TGF)- $\beta$ and platelet-derived growth factor (PDGF)-BB interacting with their specific receptors, expressed on pericytes/MSCs, may induce myofibroblast differentiation and activation, leading to the fibrotic manifestation of disease. Furthermore, the same molecular imbalance leads on one hand to pericyte proliferation and consequent wall remodeling in arteriolar vessels, on the other to pericyte detachment in capillaries. Furthermore, pericyte hyper-production of vascular endothelial growth factor (VEGF)-A may make ECs unresponsive to the proangiogenic stimuli of the tissue-derived form of VEGF-A. The increased expression of VEGFR2 on pericytes/MSCs may suppress PDGFR signaling through the induction of VEGFR2/PDGFR complexes. This pathway abrogates pericyte coverage and leads to vascular instability and regression. These alterations underlie the main pathogenic aspects of the SSc vasculopathy and the impaired compensatory neo-angiogenesis. 
myofibroblasts, thus, linking the vascular damage to some fibrotic events underling the development and progression of SSc.

\section{Conclusions}

Although our descriptive observational study does not directly address the mechanism of EC/MSC crosstalk, it gives us much information on the modulation and production of molecules involved in the pathogenesis of SSc, which may be targeted in future studies and suggests that a better understanding of the interplay between ECs and perivascular MSCs might open new future possibilities for regenerative medicine, targeting the early steps of fibrosis, for which convincing therapies are not presently available.

\footnotetext{
Abbreviations

Ang1/2: angiopoietin1/2; BM: Bone marrow; Col1: collagen1;

DMEM: Dulbecco's modified Eagle's medium; ECs: endothelial cells;

ELISA: enzyme-linked immunosorbent assay; FBS: fetal bovine serum; HC: healthy control; MSC: mesenchymal stem cell; PBS: phosphate-buffered saline; PDGF: platelet-derived growth factor; SSc: systemic sclerosis; TGF- $\beta$ : transforming growth factor $\beta$; VEGF: vascular endothelial growth factor; aSMA: alpha-smooth muscle actin
}

\section{Competing interests}

The authors declared that they have no competing interest.

\section{Authors' contributions}

PC: study conception and design, data interpretation, literature search, figure creation, writing, paper revision and acceptance; PDB: study conception and design, data interpretation, literature search, figure creation, writing, paper revision and acceptance; PR: data collection, data interpretation, literature search, paper revision and acceptance; AC: data collection, data interpretation, literature search, paper revision and acceptance; VL: data collection, literature search, paper revision and acceptance; FC: data collection, data interpretation, literature search, paper revision and acceptance; IP: data collection, literature search, paper revision and acceptance; OB: data collection, literature search, paper revision and acceptance; IS: data collection, data interpretation, literature search, paper revision and acceptance; RG: study design, data interpretation, writing, paper revision and acceptance. All authors gave final approval for submitting the manuscript for review and agree to be accountable for all aspects of the work.

\section{Acknowledgements}

The authors thank Dr Maria Paola Nanni Costa and Dr Samuele Di Giovanni for their contribution in BM aspiration. The authors thank Mrs Federica Sensini for her technical assistance. This work was supported by Fondazione Italiana Ricerca per l'Artrite (FIRA) 2009. Written informed consent was obtained from the patients or their relatives for publication of this manuscript and accompanying images.

\section{Author details \\ 'Department of Applied Clinical Sciences and Biotechnology, Rheumatology Unit, School of Medicine, University of L'Aquila, Delta 6 Building, Via dell'Ospedale, 67100 L'Aquila, Italy. ${ }^{2}$ Department of Molecular Medicine, School of Medicine 'Sapienza' University of Rome, Viale Regina Elena, 324, 00161 Rome, Italy.}

Received: 7 March 2014 Accepted: 28 August 2014

Published online: 24 September 2014

\section{References}

1. Varga J, Abraham D: Systemic sclerosis: a prototypic multisystem fibrotic disorder. J Clin Invest 2007, 117:557-567.

2. LeRoy EC: Systemic sclerosis. A vascular perspective. Rheum Dis Clin North Am 1996, 22:675-694.
3. Loeys BL, Gerber EE, Riegert-Johnson D, labal S, Whiteman P, McConnell V, Chillakuri CR, Macaya D, Coucke PJ, De Paepe A, Judge DP, Wigley F, Davis EC, Mardon HJ, Handford P, Keene DR, Sakai LY, Dietz HC: Mutations in fibrillin-1 cause congenital scleroderma: stiff skin syndrome. Sci Transl Med 2010, 2:23ra20.

4. Dulauroy S, Di Carlo SE, Langa F, Eberl G, Peduto L: Lineage tracing and genetic ablation of ADAM12(+) perivascular cells identify a major source of profibrotic cells during acute tissue injury. Nat Med 2012, 18:1262-1270.

5. Cipriani P, Carubbi F, Liakouli V, Marrelli A, Perricone C, Perricone R, Alesse E, Giacomelli R: Stem cells in autoimmune diseases: implications for pathogenesis and future trends in therapy. Autoimmun Rev 2013, 12:709-716.

6. Hunzelmann N, Brinckmann J: What are the new milestones in the pathogenesis of systemic sclerosis? Ann Rheum Dis 2010, 69:i52-i56.

7. Carmeliet $P$, Jain RK: Molecular mechanisms and clinical applications of angiogenesis. Nature 2011, 473:298-307.

8. Gabrielli A, Avvedimento EV, Krieg T: Scleroderma. N Engl J Med 2009, 360:1989-2003.

9. Lindblom P, Gerhardt H, Liebner S, Abramsson A, Enge M, Hellstrom M, Backstrom G, Fredriksson S, Landegren U, Nystrom HC, Bergstrom G, Dejana E, Ostman A, Lindahl P, Betsholtz C: Endothelial PDGF-B retention is required for proper investment of pericytes in the microvessel wall. Genes Dev 2003, 17:1835-1840.

10. Antonelli-Orlidge A, Saunders KB, Smith SR, D'Amore PA: An activated form of transforming growth factor beta is produced by cocultures of endothelial cells and pericytes. Proc Natl Acad Sci USA 1989. 86:4544-4548

11. Hirschi KK, Rohovsky SA, D'Amore PA: PDGF, TGF-beta, and heterotypic cell-cell interactions mediate endothelial cell-induced recruitment of $10 \mathrm{~T} 1 / 2$ cells and their differentiation to a smooth muscle fate. J Cell Biol 1998, 141:805-814.

12. Armulik A, Abramsson A, Betsholtz C: Endothelial/ pericyte interactions Circ Res 2005, 97:512-523.

13. Diaz-Flores L, Gutierrez R, Gonzalez P, Varela H: Inducible perivascular cells contribute to the neochondrogenesis in grafted perichondrium. Anat Rec 1991, 229:1-8.

14. Díaz-Flores L, Gutierrez R, Lopez-Alonso A, Gonzalez R, Varela H: Pericytes as a supplementary source of osteoblasts in periosteal osteogenesis. Clin Orthop Relat Res 1992, 275:280-286.

15. Saleh FA, Whyte M, Ashton P, Genever PG: Regulation of mesenchymal stem cell activity by endothelial cells. Stem Cells Dev 2011, 20:391-403.

16. Díaz-Flores L, Gutiérrez R, Madrid JF, Varela H, Valladares F, Acosta E, Martín-Vasallo P, Díaz-Flores L Jr: Pericytes. Morphofunction, interactions and pathology in a quiescent and activated mesenchymal cell niche. Histol Histopathol 2009, 24:909-969.

17. Dulmovits BM, Herman IM: Microvascular remodeling and wound healing: A role for pericytes. Int J Biochem Cell Biol 2012, 44:1800-1812.

18. Humphreys BD, Lin SL, Kobayashi A, Hudson TE, Nowlin BT, Bonventre JV, Valerius MT, McMahon AP, Duffield JS: Fate tracing reveals the pericyte and not epithelial origin of myofibroblasts in kidney fibrosis. Am J Pathol 2010, 176:85-97.

19. Göritz C, Dias DO, Tomilin N, Barbacid M, Shupliakov O, Frisén J: A pericyte origin of spinal cord scar tissue. Science 2011, 333:238-242.

20. Sponheim J, Pollheimer J, Olsen T, Balogh J, Hammarström C, Loos T, Kasprzycka M, Sørensen DR, Nilsen HR, Küchler AM, Vatn MH, Haraldsen G: Inflammatory bowel disease-associated interleukin-33 is preferentially expressed in ulceration-associated myofibroblasts. Am J Pathol 2010, 177:2804-2815.

21. Sato M, Suzuki S, Senoo H: Hepatic stellate cells: unique characteristics in cell biology and phenotype. Cell Struct Funct 2003, 28:105-112.

22. Cai X, Lin Y, Friedrich CC, Neville C, Pomerantseva I, Sundback CA, Zhang Z, Vacanti JP, Hauschka PV, Grottkau BE: Bone marrow derived pluripotent cells are pericytes which contribute to vascularization. Stem Cell Rev 2009, 5:437-445.

23. Bianco P, Riminucci M, Gronthos $S$, Robey PG: Bone marrow stromal stem cells: nature, biology, and potential applications. Stem Cells 2001, 19:180-192.

24. Cipriani P, Marrelli A, Di Benedetto P, Liakouli V, Carubbi F, Ruscitti P, Alvaro S, Pantano I, Campese AF, Grazioli P, Screpanti I, Giacomelli R: Sclerodermia mesenchymal stem cells display a differentphenotype from healthy 
controls; implications for regenerative medicine. Angiogenesis 2013, 16:595-607.

25. Pedersen $T O$, Blois $A L$, Xue $Y$, Xing $Z$, Sun $Y$, Finne-Wistrand $A$, Lorens JB, Fristad I, Leknes KN, Mustafa K: Mesenchymal stem cells induce endothelial cell quiescence and promote capillary formation. Stem Cell Res Ther 2014, 5:23.

26. Bergers $\mathrm{G}$, Song $\mathrm{S}$ : The role of pericytes in blood-vessel formation and maintenance. Neuro Oncol 2005, 7:452-464.

27. LeRoy EC, Black C, Fleischmajer R, Jablonska S, Krieg T, Medsger TA Jr, Rowell N, Wollheim F: Scleroderma (systemic sclerosis): classification, subsets and pathogenesis. J Rheumatol 1988, 15:202-205.

28. Cipriani P, Guiducci S, Miniati I, Cinelli M, Urbani S, Marrelli A, Dolo V, Pavan A, Saccardi R, Tyndall A, Giacomelli R, Cerinic MM: Impairment of endothelial cell differentiation from bone marrow-derived mesenchymal stem cells: new insight into the pathogenesis of systemic sclerosis. Arthritis Rheum 2007, 56:1994-2004.

29. Lilly B, Kennard S: Differential gene expression in a coculture model of angiogenesis reveals modulation of select pathways and a role for Notch signaling. Physiol Genomics 2009, 36:69-78.

30. Liu H, Kennard S, Lilly B: NOTCH3 expression is induced in mural cells through an autoregulatory loop that requires endothelial-expressed JAGGED1. Circ Res 2009, 104:466-475.

31. Lin SL, Chang FC, Schrimpf C, Chen YT, Wu CF, Wu VC, Chiang WC, Kuhnert F, Kuo CJ, Chen YM, Wu KD, Tsai TJ, Duffield JS: Targeting endothelium-pericyte cross talk by inhibiting VEGF receptor signaling attenuates kidney microvascular rarefaction and fibrosis. Am J Pathol 2011, 178:911-923.

32. Jimenez SA: Role of endothelial to mesenchymal transition in the pathogenesis of the vascular alterations in systemic sclerosis. ISRN Rheumatol 2013, 2013:1-15.

33. Evensen L, Micklem DR, Blois A, Berge SV, Aarsaether N, Littlewood-Evans A, Wood J, Lorens JB: Mural cell associated VEGF is required for organotypic vessel formation. PLoS One 2009, 4:e5798.

34. Darland DC, Massingham L, Smith SR, Piek E, Saint-Geniez M, D'Amore PA: Pericyte production of cell-associated VEGF is differentiation-dependent and is associated with endothelial survival. Dev Biol 2003, 264:275-288.

35. Greenberg JI, Shields DJ, Barillas SG, Acevedo LM, Murphy E, Huang J, Scheppke L, Stockmann C, Johnson RS, Angle N, Cheresh DA: A role for VEGF as a negative regulator of pericyte function and vessel maturation. Nature 2008, 456:809-813.

36. Manetti M, Guiducci S, Romano E, Bellando-Randone S, Lepri G, Bruni C, Conforti ML, Ibba-Manneschi L, Matucci-Cerinic M: Increased plasma levels of the VEGF165b splice variant are associated with the severity of nailfold capillary loss in systemic sclerosis. Ann Rheum Dis 2013, 72:1425-1427.

37. Harris S, Craze M, Newton J, Fisher M, Shima DT, Tozer GM, Kanthou C: Do anti-angiogenic VEGF (VEGFxxxb) isoforms exist? A cautionary tale. PLoS One 2012, 7:e35231

38. Harris S, Craze M, Newton J, Fisher M, Shima DT, Tozer GM, Kanthou C: VEGF121b and VEGF165b are weakly angiogenic isoforms of VEGF-A. Mol Cancer 2010, 31:320.

39. Kisseleva T, Cong M, Paik Y, Scholten D, Jiang C, Benner C: Myofibroblasts revert to an inactive phenotype during regression of liver fibrosis. Proc Natl Acad Sci 2012, 109:9448-9453.

40. Gilbane AJ, Denton CP, Holmes AM: Scleroderma pathogenesis: a pivotal role for fibroblasts as effector cells. Arthritis Res Ther 2013, 15:215.

41. Rodriguez A, Friman T, Kowanetz M, van Wieringen T, Gustafsson R, Sundberg C: Phenotypical differences in connective tissue cells emerging from microvascularpericytes in response to overexpression of PDGF-B and TGF- $\beta 1$ in normal skin in vivo. Am J Pathol 2013, 182:2132-2146.

42. Goumans MJ, Liu Z, ten Dijke P: TGF-b signaling in vascular biology and dysfunction. Cell Res 2009, 19:116-127.
43. Yamakage A, Kikuchi K, Smith EA, LeRoy EC, Trojanowska M: Selective upregulation of platelet-derived growth factor alpha receptors by transforming growth factor beta in scleroderma fibroblasts. J Exp Med 1992, 175:1227-1234.

44. Rajkumar C, Sundberg VS, Abraham DJ, Rubin K, Black CM: Activation of microvascular pericytes in autoimmune Raynaud's phenomenon and systemic sclerosis. Arthritis Rheum 1999, 42:930-941.

doi:10.1186/s13075-014-0442-z

Cite this article as: Cipriani et al:: Impaired Endothelium-Mesenchymal Stem Cells Cross-talk in Systemic Sclerosis: a Link Between Vascular and Fibrotic Features. Arthritis Research \& Therapy 2014 16:442.

\section{Submit your next manuscript to BioMed Central and take full advantage of:}

- Convenient online submission

- Thorough peer review

- No space constraints or color figure charges

- Immediate publication on acceptance

- Inclusion in PubMed, CAS, Scopus and Google Scholar

- Research which is freely available for redistribution

Submit your manuscript at www.biomedcentral.com/submit
( BioMed Central 